\title{
UNA PROPUESTA CONCEPTUAL-TEÓRICA PARA ENTENDER LAS MEDIDAS EN MATERIA DE POBLACIÓN EN COSTA RICA. SEGUNDA MITAD DEL SIGLO XX
}

\section{A CONCEPTUAL-THEORETICAL PROPOSAL TO UNDERSTAND POPULATION MEASURES IN COSTA RICA. SECOND HALF OF THE 20TH CENTURY}

\author{
Natalia Carballo Murillo*
}

RESUMEN

El objetivo de este artículo es proponer un marco conceptual y teórico para entender las percepciones, las actitudes y los tipos de masculinidades como aspectos que pueden presentarse entre la población y la adopción de medidas en materia de población en Costa Rica durante la segunda mitad del siglo xx. La construcción de la propuesta se hizo a partir de la revisión de bibliografía secundaria. La principal reflexión es, a pesar de que en Costa Rica no hubo una política con objetivos específicos para controlar la natalidad, si se dio la creación de una serie de instituciones con objetivos en materia de población. En el actuar individual ante dichas instituciones y objetivos, mediado por el contexto y por el impulso del Estado (de todo el aparato desarrollado entre las instituciones nacionales e internacionales) se ponen en evidencia las percepciones, actitudes y roles de género, así como la influencia de actores como la iglesia católica.

PALABRAS CLAVE: POBLACIÓN * PLANIFICACIÓN DE LA FAMILIA * PERCEPCIÓN * MASCULINIDADES * ACTITUD

\section{ABSTRACT}

The objective of this article is to propose a conceptual and theoretical framework to understand the perceptions, attitudes and types of masculinities, as present aspects among the population and the adoption, or not, of the population measures in Costa Rica during the second middle of the 20th century. The construction of the proposal

Centro de Información y Referencia sobre Centroamérica y el Caribe (CIRCA) del Centro de Investigación en Identidad y Cultura Latinoamericanas (CIICLA), Universidad de Costa Rica, Sede del Pacífico, Puntarenas, Costa Rica. natalia.carballomurillo@ucr.ac.cr/natalia.carballo.m@gmail.com 
was made from the review of secondary bibliography. The main reflection is, despite the fact that in Costa Rica there was no policy with specific objectives to control the birth rate, there was the creation of a series of institutions with population objectives. In individual action in the face of these institutions and objectives, mediated by the context and the impulse of the State (of the entire apparatus developed between national and international institutions), gender perceptions, attitudes and roles, as well as the influence of actors like the catholic church.

KEYWORDS: POPULATION * FAMILY PLANNING * PERCEPTION * MASCULINITIES * ATTITUDES

\section{INTRODUCCIÓN Y ANTECEDENTES}

Los estudios de Luis Rosero ${ }^{1}$ sobre la dinámica de la población, dinámica demográfica, de María Carranza ${ }^{2}$ y Mayra Cartín ${ }^{3}$ sobre planificación familiar, solo por mencionar algunas investigadoras e investigadores, así como los estudios de instituciones, por ejemplo, los Seminarios Nacionales de Demografía auspiciados por la Dirección General de Estadísticas y Censos, entre otros, se han publicado desde los años 70-80. Basta con visitar la biblioteca del Centro Centroamericano de Población de la Universidad de Costa Rica ${ }^{4}$ para darse cuenta de las distintas investigaciones.

En ese sentido este artículo tiene como objetivo proponer un marco conceptual y teórico para entender las percepciones, las actitudes y los tipos de masculinidades, como aspectos que podrían presentarse o no entre la población $y$ la adopción de medidas en materia de población en Costa Rica, a través de la actuación del Estado. Se busca contestar la pregunta ¿cómo entender el actuar público ante el contexto internacional $y$ la posterior puesta en marcha

1 Las distintas publicaciones de Luis Rosero se pueden consultar en: https://roserobixby.com/ Publicaciones.html

Por ejemplo: link.gale.com/apps/doc/A323259216/ IFME? $\mathrm{u}=$ anon $\sim \mathrm{a} 8 \mathrm{c} 840 \mathrm{f \& sid}=$ googleScholar\&xid= $4 \mathrm{ec} 00388$.

El proyecto "Personajes de la Planificación Familiar en Costa Rica" se puede consultar en: https://ccp.ucr.ac.cr/proyectos/planificacion-familiar

Por ejemplo: https://www.scielo.sa.cr/ scielo.php?script=sci_arttext\&pid $=$ S1409-14292013000200013

4 de iniciativas en materia de población en Costa Rica? Asimismo, se busca ofrecer un enfoque diferente para estudiar dichas medidas, que de seguro servirá para entender cómo se gestan y discuten en la actualidad políticas relacionadas con la población.

Para ello, el ensayo se dividirá en dos secciones. En la primera, se hará un breve repaso del contexto internacional de las políticas de población, se reconoce que el contexto internacional tuvo influencia sobre las distintas respuestas de los países al momento de implementar políticas y medidas en materia de población. En la segunda parte se describirá la propuesta teórico-conceptual.

Con el cambio del contexto y las condiciones económicas y políticas internacionales que trajo consigo la Segunda Guerra Mundial, se transforma la idea sobre la relación desarrollo-fecundidad. Como lo explica Julio Pérez:

Si hasta el momento todos los estudiosos coincidían en que el desarrollo económico era el desencadenante de la transición y la causa del descenso final de la fecundidad, se iba a sostener ahora que el descenso de la fecundidad era una condición necesaria para el desarrollo, ya que el rápido crecimiento poblacional impedía la acumulación de capital imprescindible para el despegue industrial. (Pérez, 1994, p. 6).

La "explosión demográfica en los países pobres del mundo ... despertó por primera vez una grave preocupación internacional" (Hobsbawm, 1995, p. 347). Dicha explosión del mundo se explicó por medio de la teoría de la 
transición demográfica, de un estadio de altas tasas de natalidad y mortalidad se pasa a un estadio de altas tasas de natalidad, pero con una disminución de las tasas de mortalidad por lo que aumentan las poblaciones.

Algunos países con poblaciones gigantescas estaban tan preocupados por las decenas de millones de nuevas bocas que había que alimentar cada año, que de vez en cuando sus gobiernos emprendían campañas de coacción despiadada para imponer el control de la natalidad o algún tipo de planificación familiar a sus ciudadanos. (Hobsbawm, 1995, p. 348).

Para el caso de América Latina, Susana Torrado (1997) explica:

A fines de los años 60 - como directa consecuencia de la nueva dinámica iniciada en la segunda postguerra $y$ de su eventual impacto sobre los objetivos del desarrollo- la dimensión demográfica de la sociedad emerge en América Latina como un "problema". Surge entonces la idea de que es preciso promover "soluciones" al mismo, las que necesariamente revisten la forma de políticas públicas (= acción del Estado) encaminadas a modificar el curso previsto de aquel impacto para facilitar el logro de esos objetivos. (p. 7).

En ese contexto mundial y latinoamericano se hace uso de dos teorías para tratar de explicar, entender y tomar acción. Por un lado, la teoría de la primera transición demográfica que se mencionó anteriormente:

... que trata de explicar el proceso por medio del cual bajan, en un primer momento, de manera significativa las tasas de mortalidad, dando como resultado un rápido y vertiginoso crecimiento de la población; $y$ en un segundo momento, las tasas de natalidad, produciendo estabilidad en su crecimiento y en sus respectivas tasas. (Carballo y Bartels, 2014, p. 23).
Por el otro, la teoría neomalthusiana que explica la relación entre crecimiento demográfico y pobreza bajo la cobija del imperialismo. Las ideas de Malthus se revitalizaron y dieron origen a una corriente de pensamiento denominado neomaltusianismo, que preconizaba el control de la natalidad en los países subdesarrollados. Esta surge en una época en la cual se realizaban diversos esfuerzos para solucionar la situación de crisis económica y social. Entre las soluciones propuestas y las políticas perseguidas estaba el control de la natalidad, como una respuesta a la miseria, el hambre, $y$, además, como una medida preventiva a futuros conflictos sociales desestabilizadores del sistema político vigente. Entre los precursores de esta corriente de pensamiento se encontraban a nivel internacional tres tipos de agentes: a) fundaciones privadas, b) organismos internacionales y c) gobiernos de países desarrollados (Consuegra, 1969). Con esa ideología como referente, estos agentes concretaron dos acciones relacionadas entre sí, el estudio de la situación y la dinámica demográfica de los países considerados en vías de desarrollo o del tercer mundo, $y$ el financiamiento de programas dirigidos al control de la natalidad y la planificación familiar en dichos países.

Durante la segunda mitad del siglo xx, el tema de la población fue importante $y$ entendido desde la fecundidad, es decir, desde la cantidad de nacimientos que se traducía en la cantidad de población, o desde la amenaza que para los países desarrollados y ricos representaban los países considerados en vías de desarrollo o pobres, con grandes poblaciones y en crecimiento.

El tema estuvo en las agendas económicas y políticas de los países del primer mundo como Canadá, Suiza y Estados Unidos, materializándose por medio de donaciones, financiamiento, apoyo técnico, entre otros, para la creación y puesta en marcha de una serie de medidas con el fin de moderar el crecimiento de la población de las naciones que por su alta fecundidad podrían representar una amenaza. Así como en países en vías de desarrollo, entre ellos, Costa Rica, en donde se fomentaron espacios de discusión en torno a las implicaciones 
de un exponencial crecimiento de la población sobre la economía y el desarrollo. Las políticas de población se explican, entonces, desde un escenario internacional $y$ vertical, de organizaciones externas hacia los gobiernos nacionales y de ahí a la población.

Según la investigación de Mary Lisbeth González, "la primera fundación que investigó acerca de los problemas de la población fue la Scripp para la investigación de los problemas demográficos, que se creó en 1922" (González, 1985 , p. 42). Otros institutos fundados fueron la "Office of Population Research" de 1936 y el "Population Research Center" de 1945, ambos de origen universitario. Pero, sí de injerencia política y económica en materia de población se habla, es preciso mencionar a la Fundación Rockefeller, la Fundación Ford, el Consejo de Población, la International Planned Parenthood Federation (IPPF), la Comisión de Población y la División de Población de las Naciones Unidas y del Fondo de las Naciones Unidas para Actividades en Materia de Población (UNFPA), constituido en 1969 "como organismo especializado en los problemas demográficos" (González, 1985, p. 56). Algunas de ellas con presencia en el país.

En Costa Rica no existió una política de población que buscara de forma explícita modificar la dinámica demográfica. Atendiendo a un población conservadora y religiosa, las medidas en materia de población se escudaron en el bienestar de la familia y la educación sexual, como explica Luis Rosero en 1981, "el país nada ha hecho aún en materia de políticas de población ... quienes sostienen que sí confunden a esta con políticas en lo económico y social o con la existencia de un vigoroso programa de planificación familiar" (Rosero, 1981, p. 135).

Sin embargo, la inexistencia de una política de población no significó que no existiera apoyo financiero $y$ vínculos con instituciones internacionales para aspectos relacionados. Evidencia de ello, durante la segunda mitad del siglo xx hubo un interés por parte de distintas instituciones nacionales de estudiar la dinámica poblacional, con apoyo en algunos casos de financiamiento de instituciones internacionales. Por ejemplo, el cambio en la fecundidad y sus determinantes se explicó desde distintas disciplinas, $y$ desde el momento en que empieza a ser evidente. La primera encuesta de reproducción para el área metropolitana se realizó en 1964 y, en 1969, se realiza la primera para el área rural. Asimismo, se realizaron: la Encuesta Nacional de Fecundidad de 1976, la Encuesta de Prevalencia Anticonceptiva de 1981, la Encuesta de Fecundidad y Salud, de 1986 y la Encuesta Nacional de Salud Reproductiva de 1993.

De igual manera se crearon espacios como los Seminarios Nacionales de Demografía auspiciados por la Dirección General de Estadísticas y Censos para dar a conocer trabajos de investigación demográfica y propiciar el intercambio de ideas entre representantes de diversas instituciones asociadas al campo de la demografía. En total se celebraron nueve en los años: 1957, 1958, 1961, 1968, 1970, 1976, 1979, 1983 y 1984.

A través de la presentación de trabajos sobre población, se buscaba la discusión y el intercambio de ideas sobre la situación demográfica del país y su interrelación con el desarrollo económico y social. Los expositores representaban diversos sectores y organizaciones, tanto nacionales como internacionales, como por ejemplo: la Asociación Demográfica Costarricense (ADC), la Oficina de Población, Registro Civil, el Servicio Civil, el Instituto Nacional de Vivienda y Urbanismo, el Servicio Nacional de Electricidad, el Ministerio de Trabajo, Ministerio de Agricultura, Ministerio de Educación y Ministerio de Salubridad Pública, el Movimiento Familiar Cristiano, la Universidad de Costa Rica, la Caja Costarricenses de Seguro Social (ccss), el Hospital San Juan de Dios, el Patronato Nacional de la Infancia, CELADE, la Oficina Panamericana de la Salud, la Agencia Internacional del Desarrollo y el Periódico La Nación, de Costa Rica.

También se dio la creación de asociaciones, oficinas y centros por medio de decretos de ley, relacionados con el Programa de Planificación Familiar (PFF) que inicia en 1970 como un plan piloto en cuatro clínicas periféricas de San José y en el Hospital de Turrialba (comunidad rural y alejada del centro del país). "Un año después las actividades de planificación familiar $y$ suministros de anticonceptivos se extendieron 
a todos los centros médicos del país" (González, 1985, p. 129).

Como se puede entender hay 3 actores importantes: las organizaciones internacionales, las organizaciones y ministerios nacionales, $y$ la población sobre la cual se aplicaron las medidas, incluido el Programa de Planificación Familiar.

Las organizaciones estatales creadas con objetivos en materia de población fueron: la Asociación Demográfica Costarricense (ADC, 1966), la Oficina de Población (op, 1967), el Centro de Orientación Familiar (1967), Centro de Integración Familiar (1970) y el Centro de Estudios Sociales y de la Población (1967). Los Ministerios del Estado vinculados de manera directa con la planificación familiar fueron el Ministerio de Salud y el de Educación, es de resaltar que mucho del discurso de ambos pasaba por el bienestar de la familia y la educación sexual.

El director ejecutivo de la $\mathrm{ADC} \ldots$ y el director de la oP ... propusieron crear un organismo que coordinara las actividades clínicas, educativas y de investigación en planificación familiar y educación sexual, realizadas por todas las instituciones que participaban en el PFF. La propuesta fue avalada por los miembros de las otras instituciones y se creó la Comisión Nacional de Población (CONAPO), el 30 de noviembre de 1968. (González, 1985, pp. 137-139).

Todo este proceso culminaría con la puesta en marcha del PFF en 1970. El financiamiento de las actividades de dichas organizaciones fue por donaciones de la UNFPA, la AID, la IPPF, la Fundación Ford, entre otras. La relación entre institutos nacionales, ministerios e instituciones internacionales son un ejemplo de lo que explica Carmen Miró cuando plantea:

...las decisiones que se adoptarían en ese ámbito $y$ que buscan fundamentalmente elevar el nivel y la calidad de vida de la población, nunca serían producto de una imposición unilateral por parte del Estado, sino más bien del consenso de los distintos grupos sociales. (Miró, 2001, p. 11).

En el caso de Costa Rica, las instituciones, las organizaciones y los ministerios involucrados consensuaron su participación, y su labor, según los objetivos propios de cada uno de ellos.

\section{PROPUESTA DE MARCO CONCEPTUAL- TEÓRICO}

De acuerdo a lo planteado por Miró (2001) y en la explicación de Susana Torrado (1997) sobre "que los fenómenos demográficos son el resultado, a nivel agregado, de comportamientos individuales (se sobreentiende, de individuos y familias) en los que se ponen en juego derechos humanos fundamentales" (p. 13), surge la pregunta: ¿cómo entender el actuar público ante el contexto internacional $y$ la posterior puesta en marcha de iniciativas en materia de población en Costa Rica? Para intentar responderla, en la siguiente propuesta vamos a hacer uso de conceptos y teorías relacionadas con la percepción social, las actitudes $y$ los tipos de masculinidades.

La percepción social se centra en los mecanismos de respuestas sociales $y$ de procesamiento de la información y los de representaciones sociales en los modos de conocimiento y los procesos simbólicos en su relación con la visión de mundo y la actuación social de los seres humanos. (Banchs, 1986 como se citó en Araya, 2002, p. 44).

Al tener clara la movilidad del concepto dentro del tiempo y el espacio, de acuerdo con contextos históricos y sociales, se puede decir que percibir socialmente es un:

... proceso cognitivo de la conciencia que consiste en el reconocimiento, interpretación y significación para la elaboración de juicios en torno a las sensaciones obtenidas del ambiente físico y social, en el que intervienen otros procesos psíquicos entre los que se encuentran el aprendizaje, la 
memoria y la simbolización. (Vargas, 1994, p. 48).

"En el proceso de percibir se ponen en juego referentes ideológicos y culturales que reproducen $y$ explican la realidad $y$ que son aplicados a las distintas experiencias cotidianas para ordenarlas y transformarlas." (Vargas, 1994, p. 49). También, una serie de procesos en constante interacción están de por medio, donde el individuo, la sociedad y el Estado tienen un papel activo

Lo percibido es creado con relación a las circunstancias sociales, la cultura y la clase social de pertenencia; sin embargo, la percepción real queda subjetivamente orientada hacia lo que socialmente está permitido percibir, lo cual es permeado por el Estado y para Costa Rica, en algunos casos, por la iglesia católica. Lo anterior debe ser entendido de acuerdo con la situación histórico-social, ubicada en un tiempo y espacio, que depende de circunstancias cambiantes $y$ de la adquisición de experiencias novedosas que cambian y modifican las anteriores a estas (Vargas, 1994).

La respuesta del Estado costarricense al discurso internacional hegemónico sobre el crecimiento demográfico se debió no solo a la presión de las instituciones y personas privadas y nacionales, sino también a condiciones de tipo coyuntural, sociales y políticas, que propiciaron un ambiente positivo para la adopción de las medidas y el Programa Planificación Familiar, siendo el Estado un actor importante de facilitación, así como impulsor, entre los entes internacionales $y$ nacionales $y$ la población meta, por medio de los decretos de ley que dieron origen a las instituciones nacionales que formalizaron los intereses en materia de población, y que tendrían la comunicación con las instituciones de financiamiento $y$ ayuda técnica internacional.

Pero percibir por sí solo no involucra una acción, la percepción debe ir acompañada de una actitud. El concepto de actitud contiene tres componentes: el afectivo, al encontrarse las actitudes incrustadas en la personalidad, teniendo una fuerte relación con la motivación o con la vida afectiva de las personas; el cognitivo, relacionado con las normas $y$ valores sociales que dictan la estructura del mundo y las formas de racionalidad legitimada socialmente; y la conductual, las acciones que se hagan o no siempre obedecen a una actitud (Henríquez, 2003).

De acuerdo con Sandra Araya (2002), el significado de actitud en su vínculo con las representaciones sociales es el de una "estructura particular de la orientación en la conducta de las personas cuya función es dinamizar y regular su acción." (p. 39); "expresa el aspecto más afectivo de la representación, por ser la reacción emocional acerca del objeto o del hecho." (p. 40).

Las representaciones sociales contienen a las actitudes. Una de las principales diferencias entre ambos conceptos, es que la actitud se encuentra en el polo de las respuestas. La actitud "implica la existencia de un estímulo "ya constituido" hacia el cual se reacciona según el tipo de disposición interna que se haya constituido hacia él" (Araya, 2002, p. 45), determinando la respuesta frente a un cierto estímulo.

Para Roberto Henríquez Gutiérrez, los diferentes conceptos de actitud hacen referencia a una predisposición para sentir, percibir, valorar y comportarse de una manera determinada ante un referente $\mathrm{u}$ objeto cognoscitivo, bien podría ser un conjunto de fenómenos como los grupos étnicos, instituciones, religiones, prácticas sociales, entre otros (Henríquez, 2003). Para fines de esta propuesta, la actitud o las actitudes son una respuesta ante estímulos preestablecidos, que responden a la personalidad, a las normas, las instituciones y sus leyes.

Para operacionalizar el concepto de percepción y el de actitud, primero se percibe $y$ luego se tiene la actitud, es decir, con base en las percepciones que tenga de un elemento actúo sobre ese elemento. Conceptualmente sería de la siguiente manera: se aprende referentes con los cuales se conforma evidencias, a partir de dichas evidencias las sensaciones adquieren significado al ser interpretadas e identificadas como las características de un elemento, según las sensaciones conocidas previamente. Lo anterior bajo un marco cultural e ideológico determinado en un tiempo $y$ un 
espacio, socializado del individuo con el grupo del que forma parte.

Entonces la actitud desde las tres dimensiones (afectiva, cognitiva y conductual), implica la existencia de un estímulo constituido hacia el cual se reacciona según el tipo de disposición interna, este tipo de disposición interna será la percepción de la población hacia la práctica de la anticoncepción y apertura al Programa de Planificación Familiar permeada por el papel del Estado. Pero para el caso costarricense también se debe hacer referencia a la influencia del discurso de la iglesia católica. Discurso que para la época no buscaba transgredir los roles $y$ tareas de lo considerado ser hombre o mujer, ni ninguna conducta que violentara la idea sobre "tener todos los hijos que dios quisiera" o "todos los hijos que dios mande".

Para acercarse a dicho discurso es necesario referirse al concepto de género. Para definirlo se utilizará la conceptualización de Joan Scott (1990):

El núcleo de la definición reposa sobre una conexión integral entre dos proposiciones: primero, el género como un elemento constitutivo de las relaciones sociales basadas en las diferencias que distinguen los sexos y el género es una forma primaria de relaciones significativas de poder. (p. 289).

Asimismo, "el género es el campo primario dentro del cual o por medio del cual se articula el poder" (Scott, 1990, p. 292). Dentro de la primera proposición la autora identifica cuatro elementos inter-relacionados: "símbolos culturalmente disponibles que evocan representaciones múltiples y a menudo contradictorias" (Scott, 1990, p. 289), tal como lo explica Isabel Gamboa (2000), "dentro del discurso sexual a las mujeres se les asigna un papel contradictorio: son de naturaleza pecadora pero deben ejercer la bondad." ( $p$. 81); "conceptos normativos que manifiestan las interpretaciones de los significados de los símbolos en un intento de limitar y contener sus posibilidades metafóricas." (Scott, 1990, p. 289); en el tercer aspecto incluye las "nociones políticas y referencias a las instituciones $y$ organizaciones sociales." (Scott, 1990, p. 290) y en el cuarto, la identidad subjetiva.

De los elementos anteriores, el primero está fuertemente relacionado con la idea de la mujer sumisa, obediente de dios, de su esposo, de su familia (muy característico de la época y del contexto al que se hace referencia); con respecto a la segunda, las normas establecidas por la iglesia católica, de acuerdo con lo que significa ser hombre y mujer dentro del matrimonio, relacionado con "tener los hijos que dios quiera”. El tercero cómo las relaciones laborales, la educación y las instituciones políticas influyen en el género, por ejemplo, según esta propuesta, como los tres aspectos anteriores influyeron en la mujer para tomar la decisión de planificar o no, aunque, en muchas ocasiones las instituciones sociales o las mismas organizaciones no velan por las doctrinas religiosas, sin embargo, en algunas ocasiones llegan a consensos.

Se presentan así variables constantes como las relaciones de parentesco, las normas establecidas desde el Estado y la iglesia, así como, las relaciones de poder y la lucha entre uno y otro actor, de obedecer o desobedecer, de dominar o ser dominado. El concepto de género está fuertemente ligado a lo social, las diferencias entre hombre $y$ mujer que no se pueden explicar o no se explican del todo por las características biológicas, el género las explica por medio del componente y contexto social.

Al estudiar las percepciones y actitudes de los hombres con respecto al consumo de anticonceptivos de su respuesta a las campañas de promoción del Programa de Planificación Familiar, es necesario hacer referencia a la masculinidad, o como lo plantea Mauricio Menjívar, las masculinidades. Primero, tenemos la "masculinidad hegemónica", término fuertemente vinculado a lo que la sociedad patriarcal establece sobre el hombre, la mujer y la relación entre ellos, también tiene un ligamen con las relaciones de poder entre hombres y mujeres. Según Menjívar (2001):

La importancia de las relaciones de género se refiere a que describe las relaciones de poder entre hombres y mujeres y la interiorización de tales relaciones 
partiendo, en primera instancia, de la superioridad de lo masculino y la subordinación y devaluación de los femenino y, en segunda instancia, de la dominación de unos hombres sobre otros. (p. 2).

Una de las características del modelo de la masculinidad hegemónica es su dinamismo:

Tendría que decirse que el modelo de masculinidad hegemónica no es un modelo fijo, ni "el mismo siempre y en todas partes”. Es más disputable y responde a la definición de grupos de hombres en relación con la disposición de recursos de poder económico y simbólicos, los cuales, se redefinen de un período histórico a otro. (Menjívar, 2001, p. 4).

Lo anterior ayuda a explicar por qué algunos permitieron a su esposa planificar, ir a charlas $y$ otros lo impidieron, incluso descalificándolas.

Otro tipo de masculinidad a tomar en cuenta es la masculinidad proveedora. "Existe una homologación entre la masculinidad proveedora (la cual responde ante el imperativo de proporcionar el dinero), y la paternidad." (Menjívar, 2002, p. 121). Con la aprobación de la Ley de Paternidad Responsable se devela la problemática "de que muchos hombres son progenitores sin cumplir una función paterna alguna." (Menjívar, 2002, p. 32); igualmente, conlleva a una discusión sobre la imposición de roles impuestos por la sociedad hacia el hombre.

Los casos en los cuales los hombres no cumplen con su papel de proveedor desde el punto de vista de la pensión alimenticia se deben, primero a que el hombre le da la prioridad de proveeduría a las relaciones de pareja actuales:

Al menos una buena parte de los hombres tiende a involucrarse económicamente con niños $y$ niñas acogidas en el marco de la relación de pareja en las que ellos se encuentran inmersos. Esto significa que tienden a quedar más desprotegidos los niños y niñas de relaciones de pareja anteriores, los cuales ya no parecieran encontrarse cubiertos por el término de familia en sentido estricto. (Menjívar, 2002, p. 38).

Segundo,

A la existencia de una disociación entre el ejercicio de la sexualidad y sus implicaciones sobre la reproducción, así como entre la reproducción y el ejercicio de la función paterna. Parte del resultado de esta disociación es la transferencia de las consecuencias de las propias prácticas reproductivas a la mujer, la cual "no se cuidó", muestra de un claro ejercicio irresponsable, no solo de su sexualidad, sino también de las consecuencias sobre la genitura. (Menjívar, 2002, p. 42-43).

De igual manera, "el mandato de la proveeduría tiende a desligar a los hombres de las tareas del cuido." (Menjívar, 2002, p. 48).

Los hombres relacionados con los métodos anticonceptivos, el programa de planificación familiar y otras medidas en materia de población están acogidos en una masculinidad hegemónica al mismo tiempo que proveedora. En el primer tipo se encuentran quienes no dejan planificar a su esposa, pero en el segundo caso están quienes solicitan la planificación, con lo que se evidencia una preocupación por ser padres de una familia a la que puedan proveer calidad.

El tema de las masculinidades se puede analizar desde varias perspectivas. La masculinidad hegemónica vista desde el patriarcado conlleva las siguientes consecuencias: la obligación de procrear hijos legítimos sobre la base del control político del cuerpo y sexualidad de las mujeres, mediante la creación y surgimiento de instituciones de control social (matrimonio, maternidad, virginidad, heterosexualidad obligatoria, monogamia para la mujer, entre otros) (Campos y Salas, 2002).

Con los lentes del capitalismo, Gomáriz (1997) habla del capitalismo patriarcal en el trabajo, sin embargo, el término es aplicable tanto al trabajo como a la sexualidad, la sexualidad está determinada por quien la hace, quien la controla y quien controla el producto, o sea, 
la sociedad, es decir, la sexualidad es una construcción social histórica.

Pero, a qué se hace referencia cuando se habla de masculinidad en sí. Según Álvaro Campos y José Manuel Salas alude a una manera, sobre todo en los hombres, de vivir la sexualidad, la afectividad, el trabajo, la vida diaria, de cumplir con roles sociales y sexuales y, además, a un símbolo de jerarquías sociales en el cual los varones ejercen poder sobre otros hombres, los niños y las mujeres. Por eso, se puede afirmar que la masculinidad se asocia con el poder $y$ con la autoridad (Campos y Salas, 2002).

Dentro de este concepto de masculinidad es importante rescatar la importancia del honor, como lo explica Norma Fuller, "los hombres se consideran responsables del comportamiento de sus mujeres, porque en él estriba la esencia de su honor moral y el honor moral es la esencia del honor porque está en conexión con lo sagrado." (Fuller, 1998, p. 259).

Tomando lo anterior en cuenta, cómo entender lo referente a la planificación familiar $y$ la opinión/acción del hombre, si tiene todos los hijos "que dios quiera" pero no los puede mantener no es un buen proveedor, ni buen cabeza de familia; por otro lado, si deja que su "mujer" planifique entonces está siendo afectado, ante la sociedad no puede demostrar lo buen macho que es con hijos.

La verdadera masculinidad en el aspecto sexual se prueba por la capacidad de fertilizar a la esposa, no por las hazañas sexuales. Más tarde, el auténtico hombre es aquél capaz de fundar una familia $y$ protegerla. Así, el padre suplanta al joven conquistador mientras que el macho representa solo un momento en el ciclo vital del varón. La paternidad, la capacidad de fundar una familia y protegerla frente a otros varones, no la conquista de mujeres y la lucha entre varones, son las ideales de la masculinidad lograda. (Fuller, 1998, p. 261).

Los autores coinciden en los roles de poder, dominación, paternidad y proveeduría impuestos a los hombres. A los hombres también se les exige e imponen cargas socialmente. En conclusión, la masculinidad es una forma de vivir los diferentes aspectos, el sexual, el laboral y el social, la vida de los hombres está en constante construcción y comprobación por medio de la virilidad, y la virilidad está determinada socialmente por medio de roles y estereotipos que influyen en las decisiones sobre su sexualidad $y$ en la de otras personas

\section{REFLEXIONES FINALES}

A manera de reflexión, los términos utilizados son de naturaleza dinámica, cambian de acuerdo con el contexto histórico y social, según la clase socioeconómica en la cual se está inmerso. Al cambiar el contexto cambia lo que percibe y como se actúa. Han cambiado las percepciones y con ello las actitudes, pero no así las ideas sobre los roles de género o sociales. El género, la sexualidad y la masculinidad además de ser construcciones sociales, se articulan dentro de relaciones de poder, ya sean intergenéricas, entre individuos e instituciones o entre las mismas instituciones.

A pesar de que en Costa Rica no hubo una política de población con objetivos específicos para controlar la natalidad, si se dio la creación de una serie de instituciones con objetivos en materia de población, las cuales tuvieron fuerte conexión con organizaciones privadas desde el punto de vista económico y logístico. Participar de las charlas, del Programa de Planificación Familiar, el consumir anticonceptivos, limitar los hijos, o no hacerlo, estuvo mediado por el contexto, no solo económico o político sino también social, mediado por el impulso del Estado, de todo el aparato desarrollado entre las instituciones nacionales e internacionales, como de la iglesia católica, actor que para el caso costarricense tuvo injerencia desde el pulpito en la población meta al decidir sobre las medidas en población.

Finalmente, hablar de medidas en materia de población conlleva a un ejercicio en el que se debe entender la articulación de los agentes externos con los agentes internos (Estado-iglesia), el contexto histórico-demográfico coyuntural y la clase social de pertenencia, así como estos permean las percepciones y las 
actitudes, de acuerdo con lo planteado por el género y la masculinidad.

\section{REFERENCIAS}

Araya, S. (2002). Las representaciones sociales: Ejes teóricos para su discusión. Cuaderno de Ciencias Sociales (127).

Campos, A. y Salas, J.M. (2002). Aspectos Teóricos-Conceptuales de la Masculinidad. Retos en el Siglo xxi. En A. Campos y J. Salas, Masculinidades en Centroamérica (pp. 17-51). Lara Segura Editores.

Carballo, N. y Bartels, J. (2014). (Re) Descubriendo la comarca. Punta Arenas durante el siglo xIx. Revista Estudios (28).

Consuegra, J. (1969). El Neomalthusianismo, doctrina del Neoimperialismo y análisis de las causas del subdesarrollo. Ediciones Desarrollo Indoamericano.

Fuller, N. (1998). Reflexiones sobre el machismo en América Latina. En Valdés, T. y Olavarría, J. (eds). Masculinidades y equidad de género en América Latina. FLACSO-Chile.

Gamboa, I. (2000). Los burdeles en la isla: Relaciones erótico-afectivas entre mujeres. Revista de Ciencias Sociales, II (88).

Gomáriz, E. (1997). Introducción a los estudios sobre masculinidad. Centro Nacional para el desarrollo de la Mujer y la Familia.

González, M. L. (1985). El origen y desarrollo de Planificación Familiar y el Estado: Caso de Costa Rica. Universidad de Costa Rica.
Henríquez, R. (2003). Actitudes, percepciones y valoraciones sobre paternidad de los y las estudiantes de dos colegios públicos del área metropolitana de San José. Universidad de Costa Rica.

Hobsbawm, E. (1995). Historia del Siglo XX. Crítica.

Menjívar, M. (2001). ¿Masculinidad y poder? Revista Espiga (4).

Menjívar, M. (2002). Actitudes masculinas hacia la paternidad: entre las contradicciones del mandato y el involucramiento. INAMU.

Miró, C. (2001). América Latina: políticas de población, situación demográfica y desafíos que enfrenta la región. Papeles de Población, 7 (27).

Pérez, J. (1994). La política mundial de población en el siglo xx. Papers de Demografía (90).

Rosero, L. (1981). Dinámica demográfica, planificación familiar y política de población en Costa Rica. Demografía y Economía, 15 (1).

Scott, J. W. (1990). El género: una categoría útil para el análisis histórico. En J. Amelang y M. Nash (Eds.), Historia y género: las mujeres en la Europa moderna y contemporánea. Edicions Alfons El Magnanim.

Torrado, S. (1997). Población y desarrollo: enfoques teóricos, enfoques politicos. Serie Informes de Investigación, Universidad de Buenos Aires, Facultad de Ciencias Sociales.

Vargas, L. M. (1994). Sobre el concepto de percepción. Alteridades, 8 (4).

Fecha de ingreso: 15/06/2021 Fecha de aprobación: 15/10/2021 